\title{
7 BEHÖRDEN UND KÖRPERSCHAFTEN DER ARBEITS- UND SOZIALVERWALTUNG
}

\author{
7.1 Arbeitsverwaltung
}

\subsubsection{Sondertreuhänder der Arbeit für die kulturschaffenden Berufe, Berlin \\ BDC}

Der Bestand enthält Engagementsverträge für einzelne Bühnenkünstler und Artisten (ca. 1000 Fälle, 1943-1945).

\subsubsection{Reichstreuhänder der Arbeit Schlesien, Breslau BRE, Best. 162}

Während von den entsprechenden Behörden für die Wirtschaftsgebiete Ostpreußen, Pommern, Brandenburg, Mittelelbe, Sachsen, Thüringen, Wien-Niederdonau, Oberdonau, Steiermark-Kärnten, Alpenland, Sudetenland, Danzig-Westpreußen und Wartheland kein Schriftgut erhalten blieb, dokumentiert dieser Bestand, wenn er auch nur wenige Generalakten (10 Bde, 1933-1944, darunter Dienstanweisungen, u. a. zur Bekämpfung von Arbeitsvertragsbrüchen, Geschäftsverteilungsplan, Rundschreiben des Präsidenten des Landesarbeitsamtes 1939) enthält, wie kein anderer die Praxis der Festsetzung von Löhnen und Gehältern in einzelnen Unternehmen (5048 Bde, 1933-1945, darin auch Betriebsvereinbarungen und -ordnungen und Unterlagen über die Berufung der Vertrauensleute) in folgender Gliederung:

Landwirtschaft, Gärtnerei, Tierzucht (445 Bde) - Forstwirtschaft, Fischerei (1 Bd) - Bergbau (nur Steinkohlenfabrik Breslau, 1936-1944) - Steine und Erden (64 Bde) - Eisen- und Stahlerzeugung (1 Bd) - Metallhütten und Metallhalbzeugwerke (6 Bde) - Herstellung von Eisen-, Stahl- und Metallwaren (158 Bde) - Maschinen-, Apparate- und Fahrzeugbau (177 Bde) - Elektrotechnische Industrie (82 Bde, dabei je 1 Bd betr. Siemens-Reiniger-, Siemens-Schuckert- und Siemens-Halske-Werke 1935-1945) - Chemische Industrie (73 Bde) - Textilindustrie (38 Bde) - Papierindustrie (42 Bde) - Vervielfältigungsgewerbe (79 Bde) - Leder- und Linoleumindustrie (24 Bde) - Kautschuk- und Asbestindustrie (13 Bde) - Holz- und Schnitzstoffgewerbe (50 Bde) - Musikinstrumente- und Spielwarenindustrie (1 Bd) - Nahrungsund Genußmittelgewerbe (528 Bde) - Bekleidungsgewerbe (521 Bde) - Bau- und Baunebengewerbe (395 Bde) - Wasser- und Energieversorgung und -erzeugung (2 Bde) - Reinigungsgewerbe (352 Bde) - Großhandel (420 Bde) - Einzelhandel (346 Bde) - Hilfsgewerbe des Handels (170 Bde) - Banken und Versicherungen (128 Bde) Verkehrsbetriebe (172 Bde) - Gaststättengewerbe (221 Bde) - Theater, Musik- und Sportveranstalter, Schausteller (40 Bde) - Gesundheitswesen, hygienisches Gewerbe (55 Bde).

Unter „Sonstiges“ (241 Bde) sind vor allem Akten über Löhne und Arbeitsbedingungen bei Rechtsanwälten, in Privathaushalten (Hauspersonal), von Hausmeistern und -verwaltern und in Privatschulen vereinigt.

Schriftgut des ab 1939 für Oberschlesien zuständigen Reichstreuhänders der Arbeit Kattowitz ist in der Überlieferung des Landesarbeitsamtes (vgl. unten) enthalten, jedoch nicht gesondert ausgewiesen. 


\subsubsection{Landesarbeitsämter}

Von den 14 Landesarbeitsämtern und den in den letzten Kriegsjahren an ihre Stelle tretenden Gauarbeitsämtern in Mittel- und Ostdeutschland und den eingegliederten Gebieten blieben - abgesehen von einigen Personalunterlagen des Landesarbeitsamtes Sudetenland in Reichenberg (LT, $1 \mathrm{Kt}$.) und Aktenlisten des Landesarbeitsamtes Sachsen (DD, 2 Bde, 1944) - nur folgende Bestände erhalten:

Landesarbeitsamt Mitteldeutschland, Erfurt

WE

Die überlieferten Aktenreste betreffen Personalangelegenheiten (11 Bde, 1930-1945) und enthalten Erlasse und Rundschreiben, auch des Präsidenten der Reichsanstalt für Arbeit, insbesondere zur Arbeitsmarktstatistik (15 Bde, 1928-1945) sowie acht Druckschriften (1938-1945).

Vom nachfolgenden Gauarbeitsamt Thüringen in Weimar sind Aktensplitter (WE, 1,3 lfm) überliefert, darunter Personal- (10 Bde, 1933-1946), Kassen- und Rechnungsunterlagen (4 Bde, 1945-1946) und die Druckschrift „Der Arbeitseinsatz im Gau Thüringen“. Aus dem Gauarbeitsamt Magdeburg-Anhalt in Magdeburg gibt es (MD, Rep. G 4, 2,14 lfm, 1920-1945), möglicherweise z. T. vom Reichstreuhänder der Arbeit für das Wirtschaftsgebiet Mittelelbe, Magdeburg und der Gaurechtsberatungsstelle der DAF stammende, Akten und Rundschreiben über Dienststellenverwaltung (14 Bde, 19391945), Arbeitseinsatz (3 Bde, 1943-1945), Straf- und Ordnungsstrafverfahren (4 Bde, 1942-1945), Tarife (ca. 30 Bde, 1931-1944), vom Gauarbeitsamt Halle-Merseburg in Halle ebenfalls Akten über Tarifforderungen und -verträge (ebda, 13 Bde, 1931-1938).

Landesarbeitsamt Oberschlesien, Kattowitz

KAT, Best. 129/I

Der Bestand (815 Bde, 1941-1944) enthält u. a. Rundschreiben und Anordnungen, auch des Reichsarbeitsministeriums, über Arbeitseinsatz, Entlohnung, Lebensbedingungen der Arbeitnehmer, Richtlinien zur Organisation der Arbeitsämter, Protokolle über Kontrollen in Betrieben, Schriftwechsel über Anwerbung und Zuteilung von Arbeitskräften für einzelne Berufe, Angaben zur Beschäftigungsstatistik, die Wirtschaftsstruktur der Kreise und Tarife und die von den Arbeitsämtern geförderten öffentlichen Arbeiten.

Auf Mikrofilm (BA P, Best. PL-126, 2 Filme von 17 Bden) liegen vor: Statistiken zur Arbeitseinsatzlenkung in Oberschlesien, Berichte über die sozialpolitische Entwicklung in den einzelnen Gewerbezweigen (1940-1944), über Amtsleitertagungen, Einsatz von Fremdarbeitern (mit Verzeichnissen der Durchgangslager in Oberschlesien) und Unterlagen zur Wirtschaftsstruktur einiger Arbeitsamtsbezirke.

Den Hauptteil des Bestandes (570 Bde) bilden Akten über Aufforstungsarbeiten, den Bau von Dorf- und Landstraßen (251 Bde, 1933-1936), auch Maßnahmen zum Anschluß von 56 Gemeinden an das Stromnetz (1933-1936), von Bahnhöfen und Brücken (121 Bde, 1933-1935), Arbeiten an Kanälen, zur Ent- und Bewässerung, zum Hochwasserschutz und Beseitigung von Windschäden (31 Bde, 1933-1935). Außerdem vorhanden sind Berichte über einzelne Arbeitsämter (5 Bde, 1930-1934) und Unterlagen über innere Organisation (1934-1935), Beschäftigung von Volksdeutschen, Zuteilung von Arbeitskräften (4 Bde, 1932-1940), Auszahlung von Arbeitslosengeld (2 Bde, 1937-1940), Unterstützung ausgeschiedener Wehrmachtangehörige (2 Bde, 1934-1937), Tariffragen (7 Bde, 1935-1940), Arbeitsbeschaffungsmaßnahmen im allgemeinen (10 Bde, 19321934). 


\subsubsection{Arbeitsämter}

Schriftgut von Arbeitsämtern aus der NS-Zeit ist wie in den westdeutschen Ländern nur sehr selten in staatliche Archive gelangt, vor allem solches über Arbeitsbeschaffungsmaßnahmen zwischen 1930 und 1935. Nicht selten wurden für die geringe Überlieferung Sammelbestände gebildet. Ein derartiger Bestand (BA DH) enthält vorwiegend einzelne Unterlagen zum Einsatz von Fremdarbeitern mit deren Arbeitsbüchern oder Arbeitskarten aus folgenden Arbeitsämtern: Berlin (1940-1945), Brandenburg (1943-1944), Chemnitz (1943-1945), Cottbus (1943-1945), Dresden (1935-1945), Frankfurt/Oder (1922-1943), Freiberg/Sachsen (1940-1945), Glauchau (1940-1945), Halle (1940-1945), Niederbarnim (1940-1945), Weißenfels (1942-1944).

In dem für die Arbeitsämter in Sachsen gebildeten Bestand (DD, 494 Bde, 19211945) sind mit Akten aus der NS-Zeit zu folgenden Betreffen vertreten die Arbeitsämter: - Dippoldiswalde: Arbeitsbeschaffungsmaßnahmen beim Bau von Talsperren, Straßen und Forstwegen (15 Bde, 1928-1937).

- Dresden: Stellenvermittlung des „Stahlhelm“ und der NSDAP (2 Bde, 1928-1933).

- Glauchau: Freiwilliger Arbeitsdienst (2 Bde, 1932-1934).

- Lugau: Straßen- und Wasserbau (11 Bde, 1932-1935).

- Meißen: Organisation, Besprechungen der Direktoren, Zusammenarbeit mit anderen Stellen (5 Bde, 1927-1934).

- Oelsnitz: Organisation, Konferenzen (4 Bde, 1928-1937); Arbeitsvermittlung, insbes. von Mitgliedern nationaler Wehrverbände und Ehrenzeichenträgern (6 Bde, 19331937); Landhilfe (3 Bde, 1933-1937), Anweisungen des Landesarbeitsamtes Sachsen (1932-1936); Arbeitslosenversicherung, Beiträge und Leistungen, u. a. für „SA-Kurse“ (17 Bde, 1928-1937).

- Pirna: Notstandsarbeiten im allgemeinen, Reinhardtprogramm (12 Bde, 1932-1939), Einzelmaßnahmen des Wasser- und Straßenbaus, Forstarbeiten (293 Bde, 1934-1940), Arbeitsbuch- und andere Statistik (3 Bde, 1928-1944), Stamm- und Steuerkarten (19421945) sowie Neu- und Umbau des Arbeitsamtes Auerbach (6 Bde, 1929-1941).

- Zittau: Dienststellenverwaltung, Personalangelegenheiten (31 Bde, 1928-1937); Arbeitsvermittlung (7 Bde, 1930-1937); Bildungsmaßnahmen (8 Bde, 1932-1937); Ausund Rückwanderung (3 Bde, 1928-1935); Arbeitslosenversicherung (15 Bde, 19281935); „Arbeitsschlacht“ und Arbeitsbeschaffung (7 Bde, 1930-1937, dabei 2 Bde betr. Deutschen Frauenarbeitsdienst 1933-1935), Unterbringung von Kriegsbeschädigten und Angehörigen von Wehrverbänden (4 Bde, 1934-1935).

Der entsprechende Bestand für Akten von Arbeitsämtern in Brandenburg ( $P$, Pr.Br.Rep.20B) enthält nur vom Arbeitsamt Luckenwalde eine Liste der eingesetzten Ostarbeiter und Kriegsgefangenen (1939-1945) und Restakten des Arbeitsamtes Neuruppin (28 Bde) sowie alphabetisch geordnete Personalakten von Angestellten (9 Kts).

Die meisten Aktenreste von Arbeitsämtern im Sudetenland wurden in Leitmeritz vereinigt. Sie enthalten, soweit nichts anderes angegeben, Reste von Personalakten - in der Regel nur für einzelne Anfangsbuchstaben - und stammen von den Arbeitsämtern Aussig, Freiwaldau (Uk-Stellungen 1944), Kaaden, Karlsbad (auch Übersicht über die Mitgliedschaft in Organisationen 1941), Komotau, Böhmisch Leipa, Mährisch Trübau, Reichenberg (15 Kts), Rumburg (6 Kts), Teplitz-Schönau (23 Kts, dabei auch Berichte der Abteilungsleiter Februar-April 1945, Dienstvorschriften 1939-1940, Kartei der Arbeitsbücher), Tetschen, Trautenau (11 Kts).

Daneben sind noch folgende Einzelbestände nachweisbar: 
Arbeitsamt Berlin

B, Rep. 17

Personalunterlagen von Fremdarbeitern (3 Bde, 1943-1945), Schriftwechsel des Abwehrbeauftragten (2 Bde, 1939-1945, u. a. Anweisung zur Aktenvernichtung).

Arbeitsamt Erfurt

GTH Unterlagen zur Arbeitsmarktstatistik, darunter Einsatz ausländischer Arbeitskräfte, Personalverwaltung, Haushalt und Bausachen, Runderlasse des Reichsarbeitsministeriums und des Landesarbeitsamtes Mitteldeutschland (1928-1946).

Arbeitsamt Köslin

STE, Best. 123

Akten über Frauenarbeitsdienstlager (2 Bde, 1936), Freiwilligen Arbeitsdienst, Arbeitsdienstprojekte (9 Bde, 1933-1935).

Arbeitsamt Kolberg

STE, Best. 122

Akten über Frauenarbeitsdienstlager Eickstädtswalde, Hopfenberg, Rabuhn (9 Bde, 1934-1937), Richtlinien und Projekte des Freiwilligen Arbeitsdienstes (17 Bde, 19321934).

Arbeitsamt Leslau

BRO

Personalakten - überwiegend aus dem Reich abgeordneter - Beamter und Angestellter, Unterlagen über den Abtransport von Polen aus dem Kreis Leslau zur Zwangsarbeit (1940-1944), mit Listen und Aufstellungen der Transportkosten (insgesamt 175 Bde, 1939-1945).

Arbeitsamt Litzmannstadt

LOD, Best. 37

Rundschreiben, Anordnungen (7 Bde, 1940-1944, davon 2 Bde auf Film BA P, Best: PL-152), Schriftverkehr mit dem Wehrbezirkskommando (1940-1942), Gutachten (1 Bd, 1942-1944), ärztliche Gutachten über Polen (2 Bde, 1942-1945), Zusammenarbeit mit dem Arbeitsamt Welungen (früher Wielun, 4 Bde, 1940-1944), Personalakten (3 Bde, 1939-1944), weiterer Aktenrest (GKW, Best. 718, 3 Bde, 1940-1944) mit Liste der Bediensteten, Anordnungen, Schulungsunterlagen, Werbung für die Polizeireserve.

Arbeitsamt Loben

KAT

Akten über einzelne Beschäftigte (28 Bde, 1940-1945).

Nebenstelle Patschkau des Arbeitsamtes Neiße

OP, Best. 9 Einzelne Akten (28 Bde, 1937-1944) aus fast allen Zuständigkeiten, u. a. mit Unterlagen über Zwangsarbeiter aus dem GG (1939-1941), Zusammenarbeit mit der Polizei (2 Bde, 1937-1942), Kriegsgefangene (1939-1941, u. a. aus den Lagern Lamsdorf und Gollendorf), Arbeitseinsatz im Bergbau, in der Industrie der Steine und Erden und im Buna-Werk Auschwitz-Monowitz (1941-1943), Frauenarbeitseinsatz, Heranziehung von Erbkranken (1937-1944).

Arbeitsamt Rostock

SN, Best. 2290

Akten über Meliorationsarbeiten und Freiwilligen Arbeitsdienst (17 Bde, 1929-1936).

Arbeitsamt Stendal

MD, Rep. G 4 I

Sammlung von Runderlassen (2 Bde, 1942-1945), Amtsblatt (1939). 
Arbeitsamt Troppau

TRO

Personalakten der Angestellten, Rundschreiben zur Werbung von Freiwilligen für die Luftwaffe, Militär- und Fachschulen und den öffentlichen Dienst (1941-1943), Korrespondenz über die Zuweisung und Entlassung von Arbeitskräften in Behörden, Banken, Industrie- und Landwirtschaftsbetrieben 1938-1945.

Arbeitsamt Wien

WStLA

Kataster der Arbeitslosen (Einzelfallakten 1919-1944, überwiegend bis 1938).

Arbeitsamt Zeitz

MD, Rep.G 4 I

Dienstblatt der Reichsanstalt für Arbeit (1938), Tarifunterlagen (3 Bde, 1928-1938).

\subsection{Oberversicherungsämter}

Für das Schriftgut aus der Verwaltungs- wie aus der Spruchtätigkeit der in die Bezirksregierungen eingegliederten Ämter sind selten eigene Bestände gebildet worden. Für die Oberversicherungsämter Aussig und Troppau ist nur die Angabe möglich, $\mathrm{da} ß$ von ihnen in Akten der Bezirksregierungen (vgl. oben S. 77, 79) Unterlagen vorhanden sind. Vom Oberversicherungsamt Merseburg sind in der Überlieferung der Regierung (MD, Rep. C 48 V) Generalakten über Geschäftsbetrieb (1930-1934), Personalangelegenheiten (2 Bde, 1928-1938), Amtsenthebungen aus politischen Gründen und Disziplinarsachen (17 Bde, 1931-1939) nachweisbar, vom Oberversicherungsamt Kattowitz Weisungen, Anordnungen und Berichte (1939-1943) und Einzelfallakten über Betriebe und Ärzte (580 Bde, 1940-1944). Die gesondert überlieferten Akten des Oberversicherungsamts Köslin (KÖS, Best. 36/I, 411 Bde, 1910-1940) betreffen Invaliden(80 Bde) und Unfallversicherungssachen (121 Bde) vor allem zwischen 1933 und 1936, ferner Hinterbliebenenrenten (14 Bde, 1933-1936) und Organisationsangelegenheiten (9 Bde, 1933-1934), vom Oberversicherungsamt Stettin (STE, Best. 583) reichen nur wenige allgemeine Versicherungsakten (9 Bde) bis 1933.

Überwiegend mit Vorakten des Bundesministeriums für soziale Verwaltung, dessen Kompetenzen für die Sozialversicherung ihm 1939 übertragen wurden, vereinigt ist die Überlieferung des Oberversicherungsamtes beim Reichsstatthalter in Wien (AdR, in Best. 03R102/1, ca. 85 Kts, 1938-1945). Neben der Abwicklung der österreichischen und Einführung der reichsrechtlichen Sozialversicherung, internationalen Sozialversicherungsangelegenheiten betreffen die Akten, von denen ein Teil auch aus der Spruchtätigkeit stammt, folgende Gebiete: Krankenversicherung, Aufsicht über die Kranken- und Rentenversicherungsträger, Ärzterecht, Rentenversicherung, Versicherungs- und Beitragspflicht, Personalangelegenheiten, versicherungsmathematische, statistische und versicherungstechnische Angelegenheiten, Anlegung und Verwaltung des Vermögens der Sozialversicherung, Haushalt der Versicherungsträger, Knappschaftsversicherung in der Ostmark, Sozialversicherung in der Land- und Forstwirtschaft. 


\section{3}

Versorgungsverwaltung

Nachweisbar ist lediglich vom Versorgungsamt Litzmannstadt (LO, 6060 Bde, 19391945) ein größerer Bestand von Einzelfallakten über Invaliden- und Hinterbliebenenrenten, auch mit Anträgen von Polen, vom Versorgungsamt Kattowitz ein Aktenrest (KAT, 26 Bde, 1939-1944).

\subsection{Reichsarbeitsdienst}

\subsubsection{Arbeitsgaue}

Vom Schriftgut der mehr als 20 Arbeitsgaue in Mittel- und Ostdeutschland und den eingegliederten Gebieten blieb nur wenig erhalten. Beim Arbeitsgau XV, Sachsen, handelt es sich um Aktensplitter (DD, 1934-1938) über die Abteilungen 8/151 Kotten und 8/160 Eppendorf. Aus dem Arbeitsgau XII, Oberschlesien, sind vom Meldeamt 284 in Teschen Anordnungen zur Organisation und Listen über Befreiungen von der Arbeitsdienstpflicht vorhanden (KAT, 25 Bde, 1939-1945), ein anderer Rest mit vornehmlich Werbematerial in Troppau (7 Bde, 1940-1945). Aktenreste des Arbeitsgaus II, Danzig-Westpreußen (MAW, 94 Bde, 1936-1944) sind unbearbeitet.

Ein Bestand in Wien (AdR, Best. 08R020) enthält vorwiegend Personalakten (23 Kts, 1938-1944, mit Lebensläufen, Beurteilungen, Strafverfügungen) des Aufbaustabs Österreich (1930-1939) und der Arbeitsgaue XXXIII Alpenland, Innsbruck, XXXV Oberdonau, Linz, und XXXVI Südmark, Graz, sowie des Meldeamtes Bregenz, von dem außerdem Aktenplan und Reste von Korrespondenz überliefert sind (3 Fasz., 1938-1945). Von allen genannten österreichischen Provenienzstellen sind ferner Stellenbesetzungs- und Personalveränderungslisten vorhanden (2 Kts, 4 Fasz.).

Überwiegend aus Personalunterlagen über männliches (187 Kts) und weibliches (25 Kts) Stammpersonal besteht auch die Überlieferung des Arbeitsgaus XXXVII, Sudetenland West, Teplitz-Schönau (LT, 223 Kts, 1938-1945), ferner aus Resten der Korrespondenz (1938-1939, 1943-1945), Unterlagen über disziplinarische (1942-1944) und gerichtliche Bestrafungen (1943) sowie Akten des Meldeamtes Eger (3 Kts, 19391945), u. a. über Ablehnung von Bewerbungen mit Gutachten von Ortsgruppenleitern der NSDAP mit politischen Beurteilungen.

Ersatzüberlieferung wurde im BA KO (Best. ZSg 145) gesammelt: Berichte und Materialsammlungen, z. T. aus dem Nachla $B$ des Obergeneralarbeitsführers Martin B. Eisenbach, zur Geschichte der Arbeitsgaue I, II und III, Ostpreußen (12 Bde, 1937-1978); vom Arbeitsgau IV, Pommern-Ost, Stolp, die Veröffentlichungen „Arbeitsdienst im deutschen Osten“ (1935) und „Wir vom Arbeitsgau IV“ (1939); Rundbriefe mit Chroniken und Berichten ehemaliger Angehöriger der Arbeitsgaue VIII, Brandenburg-Ost, Frankfurt/Oder, (20 Bde, 1966-1985) und X, Niederschlesien, Görlitz (1954-1975), außerdem eine Geschichte des Arbeitsgaues von Rudolf Roch u. a. in Ost-Dok. 10; die Schriften „Arbeitsdienst Sachsen-West“ des Arbeitsgaus XVI, Sachsen-West (1936), und „Unsere Thüringer Heimat im Zeichen des Arbeitsdienstes“ des Arbeitsgaus XXIII, Thüringen (um 1934), Nachkriegsberichte über den Arbeitsgau XXII, Oberschlesien (in Ost-Dok. 13). 


\subsubsection{Bezirke des Reichsarbeitsdienstes für die weibliche Jugend}

Die Überlieferung beschränkt sich auf Reste der Korrespondenz der Führerin des Bezirks XXII Südmark, Graz (AdR, in Best. 08R020, 1 Bd, 1943-1944), und Pflichtstammrollen aus 16 Landkreisen, davon sechs in Kärnten, drei in Salzburg, je zwei in Nieder- und Oberdonau und Steiermark, einer in Vorarlberg (ebda, insgesamt 5 Fasz.) und Ersatzüberlieferung in Berichten, Materialsammlungen und Publikationen für folgende Bezirke (BA KO, Best. ZSg 145, KlErw. 733): I, Ostpreußen (10 Bde, 1933-1983; Sibylle Burghardt: Chronik des weiblichen Arbeitsdienstes in Ostpreußen. Briefe, Tagebücher, Zeitungsartikel. Burgdorf 1983). - III, Mecklenburg (Material für: Lieselotte Schroeter: Geschichte und Organisation des RAD für die weibliche Jugend im Bezirk III, Mecklenburg - eine Chronik. Kiel 1979). - IV, Pommern (Gisela Schröder v. Metzsch, Irmgard v. Boehn: „Mein Herz war in Pommern.“ Witten 1980; Hildruth Schmidt-Vanderheyden: Arbeitsmaiden in Ostpommern. Ein Rückblick. Göttingen 1975). - XVI, Schlesien (Ruth Schmidt u. a.: Erinnern, besinnen, erkennen. RAD für die weibliche Jugend Bezirk 16, Niederschlesien. Kassel 1977; Liselotte Klose-Stiller: Arbeitsdienst für die weibliche Jugend in Schlesien 1930-1945. Garmisch-Partenkirchen 1978). - XXI, Oberdonau (Monatsberichte aus dem Lager Schloß Waldenfels, 2 Bde, 1939-1940). - XXVI, Wartheland (Chronik des Bezirks, 1978; Tagebuch aus dem Lager Baschkow, 1942-1943; Rosemarie Jaenchen: Arbeitsmaiden erleben das Wartheland. Berlin 1943). 
